\title{
Some congruences related to harmonic numbers and the terms of the second order sequences
}

\author{
NeŞE ÖMÜr AND Sibel KopARAL
}

\begin{abstract}
In this paper, with helps of some combinatorial identities, we investigate various basic congruences involving harmonic numbers and terms of the second order sequences $\left\{U_{k n}\right\}$ and $\left\{V_{k n}\right\}$.
\end{abstract}

\section{INTRODUCTION}

The second order sequence $\left\{W_{n}(c, d ; r, s)\right\}$, or briefly $\left\{W_{n}\right\}$, is defined for $n>0$ by

$$
W_{n+1}=r W_{n}+s W_{n-1}
$$

in which $W_{0}=c, W_{1}=d$, where $c, d, r, s$ are arbitrary integers. As some special cases of $\left\{W_{n}\right\}$, denote $W_{n}(0,1 ; r, 1), W_{n}(2, r ; r, 1)$ by $U_{n}$ and $V_{n}$, respectively.

When $r=1, U_{n}=F_{n}$ (the $n$th Fibonacci number) and $V_{n}=L_{n}$ (the $n$th Lucas number).

If $\alpha$ and $\beta$ are the roots of the equation $x^{2}-r x-1=0$, the Binet formulas of the sequences $\left\{U_{n}\right\}$ and $\left\{V_{n}\right\}$ have the forms

$$
U_{n}=\frac{\alpha^{n}-\beta^{n}}{\alpha-\beta} \quad \text { and } \quad V_{n}=\alpha^{n}+\beta^{n},
$$

respectively.

From [2, 3], E. Kılıç and P. Stanica derived the following recurrence relations for the sequences $\left\{U_{k n}\right\}$ and $\left\{V_{k n}\right\}$ for $k \geq 0, n>0$. It is clearly that

$$
\begin{aligned}
& U_{k(n+1)}=V_{k} U_{k n}+(-1)^{k+1} U_{k(n-1)}, \\
& V_{k(n+1)}=V_{k} V_{k n}+(-1)^{k+1} V_{k(n-1)},
\end{aligned}
$$

where the initial conditions of the sequences $\left\{U_{k n}\right\}$ and $\left\{V_{k n}\right\}$ are $0, U_{k}$, and $2, V_{k}$, respectively. Binet formulas of the sequences $\left\{U_{k n}\right\}$ and $\left\{V_{k n}\right\}$ are

2000 Mathematics Subject Classification. Primary: 11B39; Secondary: 11B50, 05A10, $05 \mathrm{~A} 19$.

Key words and phrases. Congruences, Harmonic numbers, Second order sequences. 
given by

$$
U_{k n}=\frac{\alpha^{k n}-\beta^{k n}}{\alpha-\beta} \quad \text { and } \quad V_{k n}=\alpha^{k n}+\beta^{k n},
$$

respectively. From the Binet formulas, one can see that $U_{-k n}=(-1)^{k n+1} U_{k n}$ and $U_{2 k n}=U_{k n} V_{k n}$. Harmonic numbers are those rational numbers given by

$$
H_{0}=0, \quad H_{n}=\sum_{k=1}^{n} \frac{1}{k}, \quad n \in \mathbb{N}=\{1,2, \ldots\} .
$$

The first few harmonic numbers are $1, \frac{3}{2}, \frac{11}{6}, \frac{25}{12}, \ldots$

For $m \in \mathbb{Z}^{+}$, harmonic numbers of order $m$ are those rational

$$
H_{n, m}=\sum_{k=1}^{n} \frac{1}{k^{m}}, \quad n \in \mathbb{N} .
$$

For a prime $p$ and an integer a with $a \nmid p$, we write the Fermat quotient $q_{p}(a)=\left(a^{p-1}-1\right) / p$. Let $\mathbb{Z}$ be the set of integers. $\mathbb{Z}_{p}$ denote the set of those rational numbers whose denominator is not divisible by $p$ and is called as the set of p-adic integer numbers. For an integer $D, \sqrt{D} \in \mathbb{Z}_{p}$ if $\left(\frac{D}{p}\right)=1$ and $\sqrt{D} \notin \mathbb{Z}_{p}$ if $\left(\frac{D}{p}\right)=-1$ in [6]. It is clearly that $x^{2}-x-1$ has two simple roots in $\mathbb{Z}_{p}$ if and only if $p \equiv \pm 1(\bmod p)$.

In [7], Z.W. Sun and L.L. Zhao established arithmetic properties of harmonic numbers. For example, for any prime $p>3$,

$$
\sum_{i=1}^{p-1} \frac{H_{i}}{i 2^{i}} \equiv \frac{7}{24} p B_{p-3} \quad\left(\bmod p^{2}\right), \quad \sum_{i=1}^{p-1} \frac{H_{i, 2}}{i 2^{i}} \equiv-\frac{3}{8} B_{p-3} \quad(\bmod p),
$$

where $B_{0}, B_{1}, B_{2}, \ldots$ Bernoulli numbers.

In [1], A. Granville showed the congruence

$$
q(x) \equiv-G(x) \quad(\bmod p), \quad p>3,
$$

where $q(x)=\frac{x^{p}-(x-1)^{p}-1}{p}$ and $G(x)=\sum_{i=1}^{p-1} \frac{x^{i}}{i}$.

In [4], H. Pan and Z. W. Sun showed the following lemma and proposition:

Lemma 1.1. Let $p>3$ be a prime. Then

$$
\begin{aligned}
\left(\frac{x^{p}+(1-x)^{p}-1}{p}\right)^{2} \equiv & -2 \sum_{i=1}^{p-1} \frac{(1-x)^{i}}{i^{2}} \\
& -2 x^{2 p} \sum_{i=1}^{p-1} \frac{\left(1-x^{-1}\right)^{i}}{i^{2}} \quad(\bmod p) .
\end{aligned}
$$


Proposition 1.1. Let $r$ and $s$ be nonzero integers. For an odd prime $p$ such that $p \nmid r s$,

$$
\begin{aligned}
\left(\frac{y_{p}-r^{p}}{p}\right)^{2} & \equiv-2 r^{2} \sum_{i=1}^{p-1} \frac{\gamma^{i}}{r^{i} i^{2}}-2 \delta^{2 p} \sum_{i=1}^{p-1} \frac{\gamma^{2 i}}{(-s)^{i} i^{2}} \quad(\bmod p), \\
\left(\frac{y_{p}-r^{p}}{p}\right)^{2} & \equiv-2 r \gamma^{p} \sum_{i=1}^{p-1} \frac{\gamma^{i}}{r^{i} i^{2}}-2 \delta^{2 p} \sum_{i=1}^{p-1} \frac{r^{i} \gamma^{i}}{s^{i} i^{2}} \quad(\bmod p),
\end{aligned}
$$

where $y_{n}=W_{n}(2, r ; r,-s)$ and $\gamma, \delta$ are the two roots of the equation $x^{2}-$ $r x+s=0$.

In this paper, we investigate the congruences involving harmonic numbers and terms of second order sequences $\left\{U_{k n}\right\}$ and $\left\{V_{k n}\right\}$. For example, for $\left(\frac{\Delta}{p}\right)=1$,

$$
\begin{aligned}
\Delta V_{k} \sum_{i=1}^{(p-1) / 2} U_{2 k(2 i+1)} H_{i} \equiv & \frac{1}{p}\left((-1)^{k}\left(V_{k}^{p} V_{k p}-\Delta^{(p+1) / 2} U_{k p}\right)-4\right) \\
& -2 q_{p}(2) V_{2 k(p+1)} \quad(\bmod p),
\end{aligned}
$$

and

$$
\sum_{i=1}^{p-1} \frac{V_{k(p+i-1)}}{V_{k}^{i}} H_{i, 2} \equiv-\frac{(-1)^{k}}{2}\left(\frac{V_{k p}-V_{k}^{p}}{p}\right)^{2} \quad(\bmod p),
$$

where $\Delta=V_{k}^{2}+4(-1)^{k+1}$, a prime number $p>3$, and an integer $k$ with $p \nmid V_{k}$.

\section{Some Lemmas}

In this section, we need the following lemmas for further use.

Lemma 2.1. For $n \in \mathbb{N}$ and $x \in \mathbb{R}$, we have the following sums:

$$
\begin{aligned}
\sum_{j=1}^{n-1} x^{j} H_{j} & =\frac{1}{1-x} \sum_{i=1}^{n-1} \frac{x^{i}}{i}-\frac{x^{n}}{1-x} H_{n-1}, \\
\sum_{j=1}^{n-1} x^{j} H_{j, 2} & =\frac{1}{1-x} \sum_{i=1}^{n-1} \frac{x^{i}}{i^{2}}-\frac{x^{n}}{1-x} H_{n-1,2} .
\end{aligned}
$$

Proof. For the proof of (5), from the sum $\sum_{i=0}^{n-1} x^{i} y^{n-i-1}=\frac{x^{n}-y^{n}}{x-y}$, we have

$$
\sum_{j=1}^{n-1} x^{j} H_{j}=\sum_{j=1}^{n-1} x^{j} \sum_{i=1}^{j} \frac{1}{i}=\sum_{i=1}^{n-1} \frac{1}{i} \sum_{j=i}^{n-1} x^{j}
$$




$$
\begin{aligned}
& =\sum_{i=1}^{n-1} \frac{1}{i}\left(\sum_{j=0}^{n-1} x^{j}-\sum_{j=0}^{i-1} x^{j}\right) \\
& =\sum_{i=1}^{n-1} \frac{1}{i}\left(\frac{1-x^{n}}{1-x}-\frac{1-x^{i}}{1-x}\right) \\
& =\sum_{i=1}^{n-1} \frac{1}{i}\left(\frac{x^{i}-x^{n}}{1-x}\right)=\frac{1}{1-x} \sum_{i=1}^{n-1} \frac{x^{i}}{i}-\frac{x^{n}}{1-x} H_{n-1},
\end{aligned}
$$

as claimed. Similarly, the other result is proven. Thus, this ends the proof.

Lemma 2.2. For $n \in \mathbb{N}$ and $x \in \mathbb{R}$, we have the following sums:

$$
\begin{aligned}
& \sum_{j=1}^{n-1} j x^{j} H_{j}= \frac{n x^{n}(x-1)-x\left(x^{n}-1\right)-x}{(x-1)^{2}} H_{n-1} \\
&-\frac{x^{n}-x}{(x-1)^{2}}+\frac{x}{(x-1)^{2}} \sum_{i=1}^{n-1} \frac{x^{i}}{i}, \\
& \sum_{j=1}^{n-1} j x^{j} H_{j, 2}=\frac{n x^{n}(x-1)-x\left(x^{n}-1\right)-x}{(x-1)^{2}} H_{n-1,2} \\
&-\frac{1}{x-1} \sum_{i=1}^{n-1} \frac{x^{i}}{i}+\frac{x}{(x-1)^{2}} \sum_{i=1}^{n-1} \frac{x^{i}}{i^{2}} .
\end{aligned}
$$

Proof. For the first claim, from the sums

$$
\sum_{i=0}^{n-1} x^{i} y^{n-i-1}=\frac{x^{n}-y^{n}}{x-y}
$$

and

$$
\sum_{i=0}^{n-1} i x^{i} y^{n-i-1}=\frac{n x^{n}(x-y)-x\left(x^{n}-y^{n}\right)}{(x-y)^{2}},
$$

we write

$$
\begin{aligned}
\sum_{j=1}^{n-1} j x^{j} H_{j} & =\sum_{j=1}^{n-1} j x^{j} \sum_{i=1}^{j} \frac{1}{i}=\sum_{i=1}^{n-1} \frac{1}{i} \sum_{j=i}^{n-1} j x^{j} \\
& =\sum_{i=1}^{n-1} \frac{1}{i}\left(\sum_{j=0}^{n-1} j x^{j}-\sum_{j=0}^{i-1} j x^{j}\right) \\
& =\sum_{i=1}^{n-1} \frac{1}{i}\left(\frac{n x^{n}(x-1)-x\left(x^{n}-1\right)}{(x-1)^{2}}\right.
\end{aligned}
$$




$$
\begin{gathered}
\left.-\frac{i x^{i}(x-1)-x\left(x^{i}-1\right)}{(x-1)^{2}}\right) \\
=\frac{n x^{n}(x-1)-x\left(x^{n}-1\right)-x}{(x-1)^{2}} H_{n-1} \\
-\frac{x^{n}-x}{(x-1)^{2}}+\frac{x}{(x-1)^{2}} \sum_{i=1}^{n-1} \frac{x^{i}}{i},
\end{gathered}
$$

as claimed. The other claim is similarly obtained. Thus, the proof is completed.

Lemma 2.3. For $n \in \mathbb{N}$ and $x \in \mathbb{R}$, we have the following sums:

$$
\begin{aligned}
\sum_{k=1}^{n-1} k^{2} x^{k} H_{k}= & \frac{x^{n}\left((n x-x-n)^{2}+x\right)}{(x-1)^{3}} H_{n-1} \\
& -\frac{n x^{n}(x-1)-3 x^{n+1}+x+2 x^{2}}{(x-1)^{3}}-\frac{x(x+1)}{(x-1)^{3}} \sum_{i=1}^{n-1} \frac{x^{i}}{i},
\end{aligned}
$$

and

$$
\begin{aligned}
\sum_{k=1}^{n-1} k^{2} x^{k} H_{k, 2}= & \frac{x^{n}\left((n x-x-n)^{2}+x\right)}{(x-1)^{3}} H_{n-1,2} \\
& -\frac{x^{n}-x}{(x-1)^{2}}+\frac{2 x}{(x-1)^{2}} \sum_{i=1}^{n-1} \frac{x^{i}}{i}-\frac{x(x+1)}{(x-1)^{3}} \sum_{i=1}^{n-1} \frac{x^{i}}{i^{2}} .
\end{aligned}
$$

Proof. Considering the sums

$$
\sum_{i=0}^{n-1} i x^{i} y^{n-i-1}=\frac{n x^{n}(x-y)-x\left(x^{n}-y^{n}\right)}{(x-y)^{2}}, \sum_{i=0}^{n-1} x^{i} y^{n-i-1}=\frac{x^{n}-y^{n}}{x-y}
$$

and

$$
\sum_{k=0}^{n-1} k^{2} x^{k} y^{n-k-1}=\frac{x^{n}\left((n x-n y-x)^{2}+x y\right)-x y^{n}(x+y)}{(x-y)^{3}},
$$

the proof is clearly given.

Lemma 2.4. Let $p$ be an odd prime. For $\left(\frac{\Delta}{p}\right)=1$,

$$
\begin{aligned}
& \text { (10) } \sum_{i=1}^{(p-1) / 2} \frac{U_{4 k i}}{i} \equiv \frac{(-1)^{k}}{p}\left(-V_{k}^{p} U_{k p}+(\sqrt{\Delta})^{p-1} V_{k p}\right) \quad(\bmod p), \\
& \text { (11) } \sum_{i=1}^{(p-1) / 2} \frac{V_{4 k i}}{i} \equiv \frac{4}{p}-\frac{(-1)^{k}}{p}\left(V_{k}^{p} V_{k p}-(\sqrt{\Delta})^{p+1} U_{k p}\right) \quad(\bmod p),
\end{aligned}
$$


where $\Delta=V_{k}^{2}+4(-1)^{k+1}$ and Legendre symbol $(\dot{\bar{p}})$.

Proof. For the proof of (11), using the Binet formula of the sequence $\left\{V_{k n}\right\}$ and taking $\frac{\alpha^{2 k}}{\beta^{2 k}}, \frac{\beta^{2 k}}{\alpha^{2 k}}$ instead of $x$ in $\sum_{i=1}^{(p-1) / 2} \frac{x^{i}}{i} \equiv \frac{2}{p}-\frac{(\sqrt{x}+1)^{p}-(\sqrt{x}-1)^{p}}{p}(\bmod p)[5]$, where any p-adic integer $x$. We get

$$
\begin{aligned}
\sum_{i=1}^{(p-1) / 2} \frac{V_{4 k i}}{i} & =\sum_{i=1}^{(p-1) / 2} \frac{\alpha^{4 k i}}{i}+\sum_{i=1}^{(p-1) / 2} \frac{\beta^{4 k i}}{i} \\
& =\sum_{i=1}^{(p-1) / 2} \frac{1}{i}\left(\frac{\alpha^{2 k i}}{\beta^{2 k i}}\right)+\sum_{i=1}^{(p-1) / 2} \frac{1}{i}\left(\frac{\beta^{2 k i}}{\alpha^{2 k i}}\right) \\
& \equiv \frac{4}{p}-\frac{V_{k}^{p}-(\sqrt{\Delta})^{p}}{p \beta^{k p}}-\frac{V_{k}^{p}-(-\sqrt{\Delta})^{p}}{p \alpha^{k p}} \\
& =\frac{4}{p}-(-1)^{k} \alpha^{k p} \frac{V_{k}^{p}-(\sqrt{\Delta})^{p}}{p}-(-1)^{k} \beta^{k p} \frac{V_{k}^{p}-(-\sqrt{\Delta})^{p}}{p} \\
& =\frac{4}{p}-\frac{V_{k}^{p}}{p}(-1)^{k}\left(\alpha^{k p}+\beta^{k p}\right)+\frac{(\sqrt{\Delta})^{p}}{p}(-1)^{k}\left(\alpha^{k p}-\beta^{k p}\right) \\
& =\frac{4}{p}-\frac{(-1)^{k}}{p}\left(V_{k}^{p} V_{k p}-(\sqrt{\Delta})^{p+1} U_{k p}\right)(\bmod p) .
\end{aligned}
$$

Similarly, using Binet formula of the sequence $\left\{U_{k n}\right\}$, the proof of the congruence in (10) is given.

Lemma 2.5. Let $p>3$ be a prime. For an integer $k$ with $p \nmid V_{k}$ and $\left(\frac{\Delta}{p}\right)=1$

$$
\sum_{i=1}^{p-1} \frac{V_{k(i+p)}}{V_{k}^{i-1} i} \equiv(-1)^{k} \frac{V_{k}^{p} V_{k(p-2)}-V_{2 k(p-1)}-(-1)^{k} V_{2 k}}{p V_{k}^{p-1}} \quad(\bmod p) .
$$

Proof. Consider

$$
\begin{aligned}
\sum_{i=1}^{p-1} \frac{V_{k(i+p)}}{V_{k}^{i-1} i} & =\sum_{i=1}^{p-1} \frac{\alpha^{k(i+p-2)}}{V_{k}^{i-1} i}+\sum_{i=1}^{p-1} \frac{\beta^{k(i+p-2)}}{V_{k}^{i-1} i} \\
& =V_{k} \alpha^{k(p-2)} \sum_{i=1}^{p-1}\left(\frac{\alpha^{k}}{V_{k}}\right)^{i} \frac{1}{i}+V_{k} \beta^{k(p-2)} \sum_{i=1}^{p-1}\left(\frac{\beta^{k}}{V_{k}}\right)^{i} \frac{1}{i} .
\end{aligned}
$$

For $\left(\frac{\Delta}{p}\right)=1$, taking $\frac{\alpha^{k}}{V_{k}}, \frac{\beta^{k}}{V_{k}}$ place of $x$ in (1), respectively, we write

$$
\sum_{i=1}^{p-1} \frac{V_{k(i+p)}}{V_{k}^{i-1} i}
$$




$$
\begin{aligned}
& \equiv V_{k} \alpha^{k(p-2)} \frac{1-\left(\frac{\alpha^{k}}{V_{k}}\right)^{p}+\left(-\frac{\beta^{k}}{V_{k}}\right)^{p}}{p}+V_{k} \beta^{k(p-2)} \frac{1-\left(\frac{\beta^{k}}{V_{k}}\right)^{p}+\left(-\frac{\alpha^{k}}{V_{k}}\right)^{p}}{p} \\
& =\frac{V_{k}^{p}\left(\alpha^{k(p-2)}+\beta^{k(p-2)}\right)-\left(\alpha^{2 k(p-1)}+\beta^{2 k(p-1)}\right)-(-1)^{k}\left(\alpha^{-2 k}+\beta^{-2 k}\right)}{p V_{k}^{p-1}} \\
& =(-1)^{k} \frac{V_{k}^{p} V_{k(p-2)}-V_{2 k(p-1)}-(-1)^{k} V_{2 k}}{p V_{k}^{p-1}}(\bmod p),
\end{aligned}
$$

as claimed.

Lemma 2.6. Let $p>3$ be a prime. For an integer $k$ with $p \nmid V_{k}$ and $\left(\frac{\Delta}{p}\right)=1$,

$$
\sum_{i=1}^{p-1} \frac{V_{k(i+p)}}{V_{k}^{i-1} i^{2}} \equiv-\frac{1}{2}\left(\frac{V_{k p}-V_{k}^{p}}{p}\right)^{2} \quad(\bmod p)
$$

Proof. Consider that

$$
\begin{aligned}
\sum_{i=1}^{p-1} \frac{V_{k(i+p)}}{V_{k}^{i-1} i^{2}} & =\sum_{i=1}^{p-1} \frac{\alpha^{k(i+p)}}{V_{k}^{i-1} i^{2}}+\sum_{i=1}^{p-1} \frac{\beta^{k(i+p)}}{V_{k}^{i-1} i^{2}} \\
& =V_{k} \sum_{i=1}^{p-1} \frac{\alpha^{k(i+p)}}{V_{k}^{i} i^{2}}+V_{k} \sum_{i=1}^{p-1} \frac{\beta^{k(i+p)}}{V_{k}^{i} i^{2}}
\end{aligned}
$$

For $\left(\frac{\Delta}{p}\right)=1$, by taking $V_{k},(-1)^{k}$ instead of $r, s$ in $(4)$, respectively, we have

$\sum_{i=1}^{p-1} \frac{V_{k(i+p)}}{V_{k}^{i-1} i^{2}} \equiv \frac{-1}{2}\left(\frac{V_{k p}-V_{k}^{p}}{p}\right)^{2}-\beta^{2 k p} \sum_{i=1}^{p-1} \frac{V_{k}^{i} \alpha^{k i}}{(-1)^{k i} i^{2}}+V_{k} \sum_{i=1}^{p-1} \frac{\beta^{k(i+p)}}{V_{k}^{i} i^{2}} \quad(\bmod p)$,

and from Fermat's little theorem, the congruence $\frac{1}{(p-k)^{2}} \equiv \frac{1}{k^{2}}(\bmod p)$ for $k \nmid p$ and $\alpha^{k} \beta^{k}=(-1)^{k}$, we get

$$
\begin{aligned}
\beta^{2 k p} \sum_{i=1}^{p-1} \frac{\left(V_{k} \alpha^{k}\right)^{i}}{(-1)^{k i} i^{2}} & =\beta^{2 k p} \sum_{i=1}^{p-1} \frac{V_{k}^{i} \alpha^{k i}}{\alpha^{k i} \beta^{k i} i^{2}}=\beta^{2 k p} \sum_{i=1}^{p-1} \frac{V_{k}^{p-i}}{\beta^{k(p-i)}(p-i)^{2}} \\
(13) \equiv \beta^{2 k p} \frac{V_{k}^{p}}{\beta^{k p}} \sum_{i=1}^{p-1} \frac{\beta^{k i}}{V_{k}^{i} i^{2}} & \equiv V_{k} \sum_{i=1}^{p-1} \frac{\beta^{k(i+p)}}{V_{k}^{i} i^{2}}(\bmod p) .
\end{aligned}
$$

By (12) and (13), we obtain the desired result. 
3. The Results involving the terms of the SeQuences $\left\{U_{k n}\right\}$ And $\left\{V_{k n}\right\}$

In this section, we give congruences for the terms of the sequences $\left\{U_{k n}\right\}$ and $\left\{V_{k n}\right\}$. Now we start with our first result.

Theorem 3.1. Let $p$ be an odd prime. For $\left(\frac{\Delta}{p}\right)=1$,

$$
\begin{gathered}
\Delta V_{k} \sum_{i=1}^{(p-1) / 2} U_{2 k(2 i+1)} H_{i} \\
(14) \equiv-\frac{4}{p}+\frac{(-1)^{k}}{p}\left(V_{k}^{p} V_{k p}-\Delta^{(p+1) / 2} U_{k p}\right)-2 q_{p}(2) V_{2 k(p+1)} \quad(\bmod p),
\end{gathered}
$$

and

$$
V_{k} \sum_{i=1}^{(p-1) / 2} V_{2 k(2 i+1)} H_{i}
$$

$$
\equiv \frac{(-1)^{k}}{p}\left(V_{k}^{p} U_{k p}-\Delta^{(p-1) / 2} V_{k p}\right)-2 q_{p}(2) U_{2 k(p+1)} \quad(\bmod p),
$$

where the Fermat quotient $q_{p}(2)=\left(2^{p-1}-1\right) / p$.

Proof. For the proof of (14), by the Binet formula of the sequence $\left\{U_{k n}\right\}$, we have

$\Delta V_{k} \sum_{i=1}^{(p-1) / 2} U_{2 k(2 i+1)} H_{i}=V_{k} \sqrt{\Delta} \sum_{i=1}^{(p-1) / 2} \alpha^{2 k(2 i+1)} H_{i}-V_{k} \sqrt{\Delta} \sum_{i=1}^{(p-1) / 2} \beta^{2 k(2 i+1)} H_{i}$.

Writing $(p+1) / 2$ place of $n$ and $\alpha^{4 k}, \beta^{4 k}$ place of $x$ in (5), respectively, we write

$$
\begin{aligned}
& \left(1-\alpha^{4 k}\right) \sum_{i=1}^{(p-1) / 2} \alpha^{4 k i} H_{i}=\sum_{i=1}^{(p-1) / 2} \frac{\alpha^{4 k i}}{i}-\alpha^{2 k(p+1)} H_{(p-1) / 2}, \\
& \left(1-\beta^{4 k}\right) \sum_{i=1}^{(p-1) / 2} \beta^{4 k i} H_{i}=\sum_{i=1}^{(p-1) / 2} \frac{\beta^{4 k i}}{i}-\beta^{2 k(p+1)} H_{(p-1) / 2} .
\end{aligned}
$$

Since $\alpha^{2 k}=\beta^{2 k} \alpha^{4 k}$ and $\beta^{2 k}=\alpha^{2 k} \beta^{4 k}$, we can rewrite

$$
\begin{aligned}
-V_{k} \sqrt{\Delta} \sum_{i=1}^{(p-1) / 2} \alpha^{4 k i+2 k} H_{i} & =\sum_{i=1}^{(p-1) / 2} \frac{\alpha^{4 k i}}{i}-\alpha^{2 k(p+1)} H_{(p-1) / 2} \\
V_{k} \sqrt{\Delta} \sum_{i=1}^{(p-1) / 2} \beta^{4 k i+2 k} H_{i} & =\sum_{i=1}^{(p-1) / 2} \frac{\beta^{4 k i}}{i}-\beta^{2 k(p+1)} H_{(p-1) / 2} .
\end{aligned}
$$


By (16) and (17), we get

$$
\begin{aligned}
& \Delta V_{k} \sum_{i=1}^{(p-1) / 2} U_{2 k(2 i+1)} H_{i} \\
= & -\sum_{i=1}^{(p-1) / 2} \frac{\alpha^{4 k i}}{i}+\alpha^{2 k(p+1)} H_{(p-1) / 2}-\sum_{i=1}^{(p-1) / 2} \frac{\beta^{4 k i}}{i}+\beta^{2 k(p+1)} H_{(p-1) / 2} \\
= & -\sum_{i=1}^{(p-1) / 2} \frac{V_{4 k i}}{i}+V_{2 k(p+1)} H_{(p-1) / 2},
\end{aligned}
$$

which, by $(11)$ and the congruence $H_{(p-1) / 2} \equiv-2 q_{p}(2)(\bmod p)$, equivalents

$$
\frac{1}{p}\left((-1)^{k}\left(V_{k}^{p} V_{k p}-(\sqrt{\Delta})^{p+1} U_{k p}\right)-4\right)-2 q_{p}(2) V_{2 k(p+1)}(\bmod p) .
$$

Similarly, using the Binet formula of the sequence $\left\{V_{k n}\right\},(16),(17),(10)$ and the congruence $H_{(p-1) / 2} \equiv-2 q_{p}(2)(\bmod p)$, the other claim is obtained.

For example, by taking $k=1$ in Teorem 3.1, for $\left(\frac{r^{2}+4}{p}\right)=1$,

$$
\begin{aligned}
& r\left(r^{2}+4\right) \sum_{i=1}^{(p-1) / 2} U_{4 i+2} H_{i} \\
\equiv & -\frac{1}{p}\left(r^{p} V_{p}-\left(r^{2}+4\right)^{(p+1) / 2} U_{p}+4\right)-2 q_{p}(2) V_{2 p+2}(\bmod p),
\end{aligned}
$$

and

$$
\begin{aligned}
& r \sum_{i=1}^{(p-1) / 2} V_{4 i+2} H_{i} \\
\equiv & -\frac{1}{p}\left(r^{p} U_{p}-\left(r^{2}+4\right)^{(p-1) / 2} V_{p}\right)-2 q_{p}(2) U_{2 p+2}(\bmod p) .
\end{aligned}
$$

Theorem 3.2. Let $p$ be an odd prime. For $\left(\frac{\Delta}{p}\right)=1$,

$$
\begin{aligned}
\Delta V_{k}^{2} \sum_{i=1}^{(p-1) / 2} i U_{4 k i} H_{i} \equiv & \left(2 U_{2 k(p+1)}-V_{k} V_{2 k p}\right) q_{p}(2)-U_{2 k(p-1)} \\
& -\frac{(-1)^{k}}{p}\left(V_{k}^{p} U_{k p}-\Delta^{(p-1) / 2} V_{k p}\right)(\bmod p),
\end{aligned}
$$

and

$$
\Delta V_{k}^{2} \sum_{i=1}^{(p-1) / 2} i V_{4 k i} H_{i} \equiv\left(2 V_{2 k(p+1)}-\Delta V_{k} U_{2 k p}\right) q_{p}(2)-V_{2 k(p-1)}+2
$$


(19)

$$
+\frac{1}{p}\left(4-(-1)^{k}\left(V_{k}^{p} V_{k p}-\Delta^{(p+1) / 2} U_{k p}\right)\right)(\bmod p),
$$

where $q_{p}(2)$ as before.

Proof. For the proof of (19), using the Binet formula of the sequence $\left\{V_{k n}\right\}$, we have

$$
\Delta V_{k}^{2} \sum_{i=1}^{(p-1) / 2} i V_{4 k i} H_{i}=\Delta V_{k}^{2} \sum_{i=1}^{(p-1) / 2} i \alpha^{4 k i} H_{i}+\Delta V_{k}^{2} \sum_{i=1}^{(p-1) / 2} i \beta^{4 k i} H_{i} .
$$

Putting $(p+1) / 2$ instead of $n$ and $\alpha^{4 k}, \beta^{4 k}$ instead of $x$ in (7), respectively, we write

$$
\begin{aligned}
& \left(\alpha^{4 k}-1\right)^{2} \sum_{i=1}^{(p-1) / 2} i \alpha^{4 k(i-1)} H_{i} \\
= & \left(\frac{p+1}{2} \alpha^{2 k(p-1)}\left(\alpha^{4 k}-1\right)-\alpha^{2 k(p+1)}\right) H_{(p-1) / 2} \\
& -\left(\alpha^{2 k(p-1)}-1\right)+\sum_{i=1}^{(p-1) / 2} \frac{\alpha^{4 k i}}{i}
\end{aligned}
$$

and

$$
\begin{aligned}
& \left(\beta^{4 k}-1\right)^{2} \sum_{i=1}^{(p-1) / 2} i \beta^{4 k(i-1)} H_{i} \\
= & \left(\frac{p+1}{2} \beta^{2 k(p-1)}\left(\beta^{4 k}-1\right)-\beta^{2 k(p+1)}\right) H_{(p-1) / 2} \\
& -\left(\beta^{2 k(p-1)}-1\right)+\sum_{i=1}^{(p-1) / 2} \frac{\beta^{4 k i}}{i} .
\end{aligned}
$$

From the equalities $\alpha^{2 k}=\beta^{2 k} \alpha^{4 k}$ and $\beta^{2 k}=\alpha^{2 k} \beta^{4 k}$, we have

$$
\begin{aligned}
\Delta V_{k}^{2} \sum_{i=1}^{(p-1) / 2} i \alpha^{4 k i} H_{i}= & \left(\frac{p+1}{2} \sqrt{\Delta} V_{k} \alpha^{2 k p}-\alpha^{2 k(p+1)}\right) H_{(p-1) / 2} \\
& -\left(\alpha^{2 k(p-1)}-1\right)+\sum_{i=1}^{(p-1) / 2} \frac{\alpha^{4 k i}}{i}, \\
\Delta V_{k}^{2} \sum_{i=1}^{(p-1) / 2} i \beta^{4 k i} H_{i}= & \left(-\frac{p+1}{2} \sqrt{\Delta} V_{k} \beta^{2 k p}-\beta^{2 k(p+1)}\right) H_{(p-1) / 2} \\
& -\left(\beta^{2 k(p-1)}-1\right)+\sum_{i=1}^{(p-1) / 2} \frac{\beta^{4 k i}}{i} .
\end{aligned}
$$


Using the Binet formulas of the sequences $\left\{U_{k n}\right\}$ and $\left\{V_{k n}\right\}$, by (20) and (21), we rewrite

$$
\begin{aligned}
& \Delta V_{k}^{2} \sum_{i=1}^{(p-1) / 2} i V_{4 k i} H_{i} \\
= & \left(\frac{p+1}{2} \sqrt{\Delta} V_{k} \alpha^{2 k p}-\alpha^{2 k(p+1)}\right) H_{(p-1) / 2}-\left(\alpha^{2 k(p-1)}-1\right) \\
& +\left(-\frac{p+1}{2} \sqrt{\Delta} V_{k} \beta^{2 k p}-\beta^{2 k(p+1)}\right) H_{(p-1) / 2}-\left(\beta^{2 k(p-1)}-1\right) \\
& +\sum_{i=1}^{(p-1) / 2} \frac{\alpha^{4 k i}}{i}+\sum_{i=1}^{(p-1) / 2} \frac{\beta^{4 k i}}{i} \\
= & \left(\frac{p+1}{2} \Delta V_{k} U_{2 k p}-V_{2 k(p+1)}\right) H_{(p-1) / 2}-V_{2 k(p-1)}+2+\sum_{i=1}^{(p-1) / 2} \frac{V_{4 k i}}{i} .
\end{aligned}
$$

From $(11)$ and the congruence $H_{(p-1) / 2} \equiv-2 q_{p}(2)(\bmod p)$, we have

$$
\begin{aligned}
\Delta V_{k}^{2} \sum_{i=1}^{(p-1) / 2} i V_{4 k i} H_{i} \equiv & \left(2 V_{2 k(p+1)}-\Delta V_{k}(p+1) U_{2 k p}\right) q_{p}(2)-V_{2 k(p-1)}+2 \\
& +\frac{4}{p}-\frac{(-1)^{k}}{p}\left(V_{k}^{p} V_{k p}-(\sqrt{\Delta})^{p+1} U_{k p}\right)(\bmod p),
\end{aligned}
$$

as claimed. Similarly, the other congruence is given. Thus, the proof is completed.

For example, when $k=r=1$ in Teorem 3.2, we have the congruences as follows: For $\left(\frac{5}{p}\right)=1$,

$$
\begin{aligned}
5 \sum_{i=1}^{(p-1) / 2} i F_{4 i} H_{i} \equiv & \left(2 F_{2 p+2}-L_{2 p}\right) q_{p}(2)-F_{2 p-2} \\
& +\frac{F_{p}-5^{(p-1) / 2} L_{p}}{p}(\bmod p),
\end{aligned}
$$

and

$$
\begin{aligned}
5 \sum_{i=1}^{(p-1) / 2} i L_{4 i} H_{i} \equiv & \left(2 L_{2 p+2}-5 F_{2 p}\right) q_{p}(2)-L_{2 p-2}+2 \\
& +\frac{L_{p}-5^{(p+1) / 2} F_{p}+4}{p}(\bmod p) .
\end{aligned}
$$


Theorem 3.3. Let $p$ be an odd prime. For $\left(\frac{\Delta}{p}\right)=1$,

$$
\begin{aligned}
& \Delta^{2} V_{k}^{3} \sum_{i=1}^{(p-1) / 2} i^{2} U_{4 k i} H_{i} \\
\equiv & -V_{2 k}\left(3+\frac{4}{p}-\frac{(-1)^{k}}{p}\left(V_{k}^{p} V_{k p}-(\sqrt{\Delta})^{p+1} U_{k p}\right)\right) \\
& +V_{2 k p}\left(3-q_{p}(2)\left(\frac{V_{4 k}}{2}+3\right)\right)-\frac{\Delta}{2} U_{2 k(p-1)} U_{2 k}(\bmod p),
\end{aligned}
$$

and

$$
\begin{aligned}
& \Delta V_{k}^{3} \sum_{i=1}^{(p-1) / 2} i^{2} V_{4 k i} H_{i} \\
\equiv & U_{2 k p}\left(3-q_{p}(2)\left(\frac{V_{4 k}}{2}+3\right)\right)-U_{2 k}\left(\frac{1}{2} V_{2 k(p-1)}+1\right) \\
& +\frac{(-1)^{k}}{p} V_{2 k}\left(V_{k}^{p} U_{k p}-(\sqrt{\Delta})^{p-1} V_{k p}\right)(\bmod p),
\end{aligned}
$$

Proof. Using the Binet formulas of the sequences $\left\{U_{k n}\right\},\left\{V_{k n}\right\}$, by (9), (10) and the congruence $H_{(p-1) / 2} \equiv-2 q_{p}(2)(\bmod p)$, we obtained the desired result.

Now, we will give the congruences with harmonic numbers of order $2, H_{n, 2}$.

Theorem 3.4. Let $p>3$ be a prime. For $\left(\frac{\Delta}{p}\right)=1$,

$$
\sum_{i=1}^{p-1} \frac{V_{k(i+p-1)}}{V_{k}^{i}} H_{i, 2} \equiv-\frac{(-1)^{k}}{2}\left(\frac{V_{k p}-V_{k}^{p}}{p}\right)^{2}(\bmod p) .
$$

Proof. From Binet formula of the sequence $\left\{V_{k n}\right\}$, we consider

$$
\begin{aligned}
& (-1)^{k} \sum_{i=1}^{p-1} \frac{V_{k(i+p-1)}}{V_{k}^{i}} H_{i, 2} \\
= & (-1)^{k} \sum_{i=1}^{p-1} \frac{\alpha^{k(i+p-1)}}{V_{k}^{i}} H_{i, 2}+\sum_{i=1}^{p-1} \frac{\beta^{k(i+p-1)}}{V_{k}^{i}} H_{i, 2} \\
= & \frac{(-1)^{k}}{\alpha^{k} V_{k}} \sum_{i=1}^{p-1} \frac{\alpha^{k(i+p)}}{V_{k}^{i-1}} H_{i, 2}+\frac{(-1)^{k}}{\beta^{k} V_{k}} \sum_{i=1}^{p-1} \frac{\beta^{k(i+p)}}{V_{k}^{i-1}} H_{i, 2} \\
= & \frac{\beta^{k}}{V_{k}} \sum_{i=1}^{p-1} \frac{\alpha^{k(i+p)}}{V_{k}^{i-1}} H_{i, 2}+\frac{\alpha^{k}}{V_{k}} \sum_{i=1}^{p-1} \frac{\beta^{k(i+p)}}{V_{k}^{i-1}} H_{i, 2} .
\end{aligned}
$$


By taking $p$ instead of $n$ and $\frac{\alpha^{k}}{V_{k}}, \frac{\beta^{k}}{V_{k}}$ instead of $x$ in (6), respectively, we have

$(22)\left(\frac{V_{k}-\alpha^{k}}{V_{k}}\right) \sum_{i=1}^{p-1}\left(\frac{\alpha^{k}}{V_{k}}\right)^{i+p} H_{i, 2}=\sum_{i=1}^{p-1} \frac{\left(\frac{\alpha^{k}}{V_{k}}\right)^{i+p}}{i^{2}}-\left(\frac{\alpha^{k}}{V_{k}}\right)^{2 p} H_{p-1,2}$,

(23) $\left(\frac{V_{k}-\beta^{k}}{V_{k}}\right) \sum_{i=1}^{p-1}\left(\frac{\beta^{k}}{V_{k}}\right)^{i+p} H_{i, 2}=\sum_{i=1}^{p-1} \frac{\left(\frac{\beta^{k}}{V_{k}}\right)^{i+p}}{i^{2}}-\left(\frac{\beta^{k}}{V_{k}}\right)^{2 p} H_{p-1,2}$.

From $(22),(23)$ and the congruence $H_{p-1,2} \equiv 0(\bmod p)$, we get

$$
(-1)^{k} \sum_{i=1}^{p-1} \frac{V_{k(i+p-1)}}{V_{k}^{i}} H_{i, 2} \equiv \sum_{i=1}^{p-1} \frac{\alpha^{k(i+p)}}{V_{k}^{i-1} i^{2}}+\sum_{i=1}^{p-1} \frac{\alpha^{k(i+p)}}{V_{k}^{i-1} i^{2}}(\bmod p) .
$$

Using Binet formula of the sequence $\left\{V_{k n}\right\}$ and Lemma 2.6, we have

$$
\begin{aligned}
(-1)^{k} \sum_{i=1}^{p-1} \frac{V_{k(i+p-1)}}{V_{k}^{i}} H_{i, 2} & \equiv \sum_{i=1}^{p-1} \frac{V_{k(i+p)}}{V_{k}^{i-1} i^{2}} \\
& \equiv-\frac{1}{2}\left(\frac{V_{k p}-V_{k}^{p}}{p}\right)^{2}(\bmod p) .
\end{aligned}
$$

which settles the proof.

Theorem 3.5. Let $p>3$ be a prime. For an integer $k$ with $p \nmid V_{k}$ and $\left(\frac{\Delta}{p}\right)=1$,

$$
\begin{aligned}
\sum_{i=1}^{p-1} i \frac{V_{k(i+p-3)}}{V_{k}^{i}} H_{i, 2} \equiv & (-1)^{k} \frac{V_{k}^{p} V_{k(p-2)}-V_{2 k(p-1)}-(-1)^{k} V_{2 k}}{p V_{k}^{p-1}} \\
& -\frac{1}{2}\left(\frac{V_{k p}-V_{k}^{p}}{p}\right)^{2}(\bmod p) .
\end{aligned}
$$

Proof. From Binet formula of the sequence $\left\{V_{k n}\right\}$ and $\alpha^{2 k} \beta^{2 k}=1$, we have

$$
\begin{aligned}
& \sum_{i=1}^{p-1} i \frac{V_{k(i+p-3)}}{V_{k}^{i}} H_{i, 2} \\
= & \sum_{i=1}^{p-1} i \frac{\alpha^{k(i+p-3)}}{V_{k}^{i}} H_{i, 2}+\sum_{i=1}^{p-1} i \frac{\beta^{k(i+p-3)}}{V_{k}^{i}} H_{i, 2} \\
= & \frac{1}{\alpha^{2 k} V_{k}^{2}} \sum_{i=1}^{p-1} i \frac{\alpha^{k(p+i-1)}}{V_{k}^{i-2}} H_{i, 2}+\frac{1}{\beta^{2 k} V_{k}} \sum_{i=1}^{p-1} i \frac{\beta^{k(i+p-1)}}{V_{k}^{i-2}} H_{i, 2} \\
= & \frac{\beta^{2 k}}{V_{k}^{2}} \sum_{i=1}^{p-1} i \frac{\alpha^{k(i+p-1)}}{V_{k}^{i-2}} H_{i, 2}+\frac{\alpha^{2 k}}{V_{k}} \sum_{i=1}^{p-1} i \frac{\beta^{k(i+p-1)}}{V_{k}^{i-2}} H_{i, 2} .
\end{aligned}
$$


If we take $p$ instead of $n$ and $\frac{\alpha^{k}}{V_{k}}, \frac{\beta^{k}}{V_{k}}$ instead of $x$ in (8), respectively, we get

$$
\begin{aligned}
& \frac{\beta^{2 k}}{V_{k}^{2}} \sum_{i=1}^{p-1} i \frac{\alpha^{k(i+p-1)}}{V_{k}^{i-2}} H_{i, 2} \\
= & V_{k}^{2} \alpha^{k(p-1)}\left(p\left(\frac{\alpha^{k}}{V_{k}}\right)^{p}\left(\frac{\alpha^{k}}{V_{k}}-1\right)-\left(\frac{\alpha^{k}}{V_{k}}\right)^{p+1}\right) H_{p-1,2} \\
& +\frac{\beta^{k}}{\alpha^{k}} \sum_{i=1}^{p-1} \frac{\alpha^{k(i+p)}}{V_{k}^{i-1} i}+\sum_{i=1}^{p-1} \frac{\alpha^{k(i+p)}}{V_{k}^{i-1} i^{2}},
\end{aligned}
$$

and

$$
\begin{aligned}
& \frac{\alpha^{2 k}}{V_{k}^{2}} \sum_{i=1}^{p-1} i \frac{\beta^{k(i+p-1)}}{V_{k}^{i-2}} H_{i, 2} \\
= & V_{k}^{2} \beta^{k(p-1)}\left(p\left(\frac{\beta^{k}}{V_{k}}\right)^{p}\left(\frac{\beta^{k}}{V_{k}}-1\right)-\left(\frac{\beta^{k}}{V_{k}}\right)^{p+1}\right) H_{p-1,2} \\
& +\frac{\alpha^{k}}{\beta^{k}} \sum_{i=1}^{p-1} \frac{\beta^{k(i+p)}}{V_{k}^{i-1} i}+\sum_{i=1}^{p-1} \frac{\beta^{k(i+p)}}{V_{k}^{i-1} i^{2}} .
\end{aligned}
$$

From $(24),(25)$ and the congruence $H_{p-1,2} \equiv 0(\bmod p)$, we have

$$
\begin{aligned}
\sum_{i=1}^{p-1} i \frac{V_{k(i+p-3)}}{V_{k}^{i}} H_{i, 2} \equiv & \frac{\beta^{k}}{\alpha^{k}} \sum_{i=1}^{p-1} \frac{\alpha^{k(i+p)}}{V_{k}^{i-1} i}+\sum_{i=1}^{p-1} \frac{\alpha^{k(i+p)}}{V_{k}^{i-1} i^{2}} \\
& +\frac{\alpha^{k}}{\beta^{k}} \sum_{i=1}^{p-1} \frac{\beta^{k(i+p)}}{V_{k}^{i-1} i}+\sum_{i=1}^{p-1} \frac{\beta^{k(i+p)}}{V_{k}^{i-1} i^{2}}(\bmod p) .
\end{aligned}
$$

By $\alpha^{k} \beta^{k}=(-1)^{k}$, we rewrite

$$
\begin{aligned}
\sum_{i=1}^{p-1} i \frac{V_{k(i+p-3)}}{V_{k}^{i}} H_{i, 2} \equiv & (-1)^{k} \sum_{i=1}^{p-1} \frac{\alpha^{k(i+p-2)}}{V_{k}^{i-1} i}+\sum_{i=1}^{p-1} \frac{\alpha^{k(i+p)}}{V_{k}^{i-1} i^{2}} \\
& +(-1)^{k} \sum_{i=1}^{p-1} \frac{\beta^{k(i+p-2)}}{V_{k}^{i-1} i}+\sum_{i=1}^{p-1} \frac{\beta^{k(i+p)}}{V_{k}^{i-1} i^{2}} \\
= & (-1)^{k} \sum_{i=1}^{p-1} \frac{V_{k(i+p-2)}}{V_{k}^{i-1} i}+\sum_{i=1}^{p-1} \frac{V_{k(i+p)}}{V_{k}^{i-1} i^{2}}(\bmod p),
\end{aligned}
$$

which, by Lemma2.5 and Lemma2.6, equivalents

$$
(-1)^{k} \frac{V_{k}^{p} V_{k(p-2)}-V_{2 k(p-1)}-(-1)^{k} V_{2 k}}{p V_{k}^{p-1}}-\frac{1}{2}\left(\frac{V_{k p}-V_{k}^{p}}{p}\right)^{2}(\bmod p) .
$$


As a result of Teorem 3.5, by taking 1 instead of $k$, we have the following corollary:

Corollary 3.1. Let $p>3$ be a prime. For $p \nmid r$, and $\left(\frac{\Delta}{p}\right)=1$,

$$
\sum_{i=1}^{p-1} i \frac{V_{i+p-3}}{r^{i}} H_{i, 2} \equiv-\frac{r^{p} V_{p-2}-V_{2 p-2}+r^{2}+2}{p r^{p-1}}-\frac{1}{2}\left(\frac{V_{p}-r^{p}}{p}\right)^{2}(\bmod p) .
$$

For example, when $r=1$ in Corollary 3.1, we have the congruence as follows:For $\left(\frac{1}{p}\right)=1$,

$$
\sum_{i=1}^{p-1} i L_{i+p-3} H_{i, 2} \equiv-\frac{L_{p-2}-L_{2 p-2}+3}{p}-\frac{1}{2}\left(\frac{L_{p}-1}{p}\right)^{2}(\bmod p) .
$$

\section{REFERENCES}

[1] A. Granville, The square of the Fermat quotient, Integers:Electron. J. Combin. Number Theory, 4 (2004), A22.

[2] E. Kılıc, P.Stanica, Factorizations and representations of second linear recurrences with indices in arithmetic progressions, Bulletin of the Mexican Mathematical Society, Vol. 15, No. 1 (2009), pp. 23-36.

[3] E. Kılıc, P.Stanica, Factorizations of binary polynomial recurrences by matrix methods, Rocky Mountain Journal of Mathematics, Vol. 41, No. 4 (2011), pp. 1247-1264.

[4] H. Pan, Z. W. Sun, Proof of three conjectures on congruences, Sci. China Math., Vol. 57, No. 10 (2014), pp. 2091-2102.

[5] S. Koparal and N. Ömür, On congruences related to Central binomial coefficients, Harmonic and Lucas Numbers, Turkish Journal of Mathematics, accepted.

[6] P.T. Young,p-adic congruences for the generalized Fibonacci sequences, The Fibonacci Quart., Vol. 32, No. 1 (1994), pp. 2-10.

[7] Z. W. Sun, L. L. Zhao, Arithmetic theory of harmonic numbers II, Colloq. Math., Vol. 130, No. 1 (2013), pp. 67-78.

NeŞE ÖMÜr

KOCAELI UNIVERSITY

Mathematics Department

41380 İzmit Kocaeli

TURKEY

E-mail address: neseomur@kocaeli.edu.tr

Sibel Koparal

KocAeli UNIVERSity

Mathematics Department

41380 İzmit KocAeli

TURKEY

E-mail address: sibel.koparal@kocaeli.edu.tr 